\title{
Analysis of Array Distortion in a Microwave Interferometric Radiometer: Application to the GeoSTAR Project
}

\author{
Francesc Torres, Senior Member, IEEE, Alan B. Tanner, Shannon T. Brown, Member, IEEE,
} and Bjorn H. Lambrigsten

\begin{abstract}
The Geostationary Synthetic Thinned Array Radiometer represents a promising new approach to microwave atmospheric sounding from geostationary orbit based on passive interferometry. Distortion due to mechanical or thermal constraints produces a displacement of the ideal antenna positions in the array that causes sampling errors. In this paper, the impact of array distortion on radiometric error is analyzed in detail so as to identify the dominant sources of error. A preliminary analysis showing that array distortion can be well corrected by means of an external phase reference is also presented.
\end{abstract}

Index Terms-Array, distortion, error analysis, interferometry, radiometry, remote sensing.

\section{INTRODUCTION}

G EOSTATIONARY microwave sounders have not yet been feasible due to the large aperture required to achieve sufficient spatial resolution. In this sense, Geostationary Synthetic Thinned Array Radiometer (GeoSTAR) represents a promising new approach to microwave atmospheric sounding from a geostationary orbit based on passive interferometry [1]. Microwave aperture synthesis was first suggested in the 1980 s as an alternative to real aperture radiometry for Earth observation with high spatial resolution [2]. The first instrument to use this concept was the Electronically Scanned Thinned Array Radiometer, which is an airborne L-band radiometer using real aperture for along-track direction and interferometric aperture synthesis for cross track [3]. A radiometer using aperture synthesis in both directions, i.e., the Microwave Imaging Radiometer by Aperture Synthesis, was proposed in [4] and [5] to provide soil moisture and ocean surface salinity global coverage measurements from space.

Manuscript received May 24, 2006; revised April 10, 2007. This work was performed in the frame of the GeoSTAR project, funded by the National Aeronautics and Space Administration, during F. Torres sabbatical stage at California Institute of Technology, Jet Propulsion Laboratory (Grant PR20050427, Spanish Ministry of Education \& Science).

F. Torres is with the Department of Signal Theory and Communications, Polytechnic University of Catalonia, 08030 Barcelona, Spain (e-mail: xtorres@tsc.upc.edu).

A. B. Tanner and B. H. Lambrigsten are with the Jet Propulsion Laboratory, California Institute of Technology, Pasadena, CA 91109 USA (e-mail: Alan.b.tanner@jpl.nasa.gov).

S. T. Brown is with the Microwave Advance Systems Section, NASA Jet Propulsion Laboratory, Pasadena, CA 91109 USA (e-mail: Shannon.T. Brown@jpl.nasa.gov).

Color versions of one or more of the figures in this paper are available online at http://ieeexplore.iee.org.

Digital Object Identifier 10.1109/TGRS.2007.898093
GeoSTAR was proposed as a solution to Geostationary Operational Environmental Satellite Systems microwave sounders in response to the 2002 NASA Research Announcement calling for proposals to develop new technologies. This instrument may complement future infrared sounders and enable all weather temperature and humidity soundings and rain mapping. While low-Earth orbit (LEO) satellites provide coverage in relative narrow swaths and with a revisit time of 12-24 h, a geostationary Earth orbit satellite (GEO) can provide continuous hemispheric or regional coverage, which makes it possible to monitor highly dynamic phenomena such as hurricanes. Sponsored by the NASA Instrument Incubator Program, the Jet Propulsion Laboratory is currently developing a proof-ofconcept ground-based demonstrator, which has already produced very encouraging results [6].

One of the major concerns about the feasibility of a geosynchronous microwave interferometric radiometer is the impact of array distortion due to mechanical and thermal constraints. This produces spatial domain sampling errors associated with the displacement of the antenna phase centers from their nominal position in the array. This paper is devoted to assessing the impact of such array distortion and evaluating the feasibility of a correction procedure.

If array distortion is present, the measured signals, which are called visibilities, are not given in a uniform grid, and Fourier techniques cannot be directly applied to simulate them. Instead, this direct case simulation (from brightness temperature to visibility) must be performed by means of the so-called G-matrix [7]. The G-matrix, which is the discrete version of the interferometric integral, allows us to estimate the measured visibilities when array distortion is present. Once these corrupted visibilities are known, the inverse case (from visibility to brightness temperature) is performed by means of an inverse Fourier transform [8]. That is, the corrupted visibilities are assigned to the nominal uniform grid to retrieve a corrupted image and assess the impact of array distortion on the radiometric error. This procedure allows the evaluation of the impact of three different types of array distortion: 1) systematic array arm misalignment; 2) array arm stretching; and 3) random antenna position errors.

\section{BASICS OF GEOSTAR}

A space version of GeoSTAR would consist of a thinned array of about 300 receiving elements deployed in a " $\mathrm{Y}$ " configuration. Since the distance between single elements is 
(a)

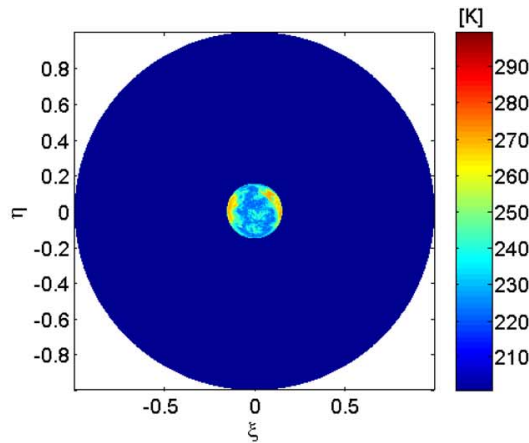

(d)

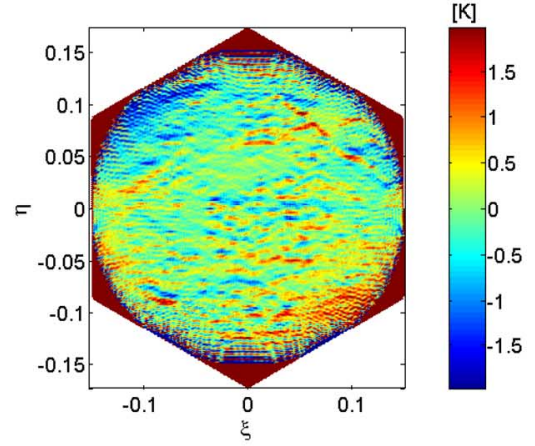

(b)

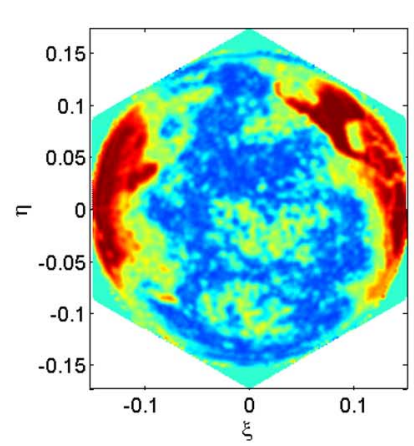

(e)
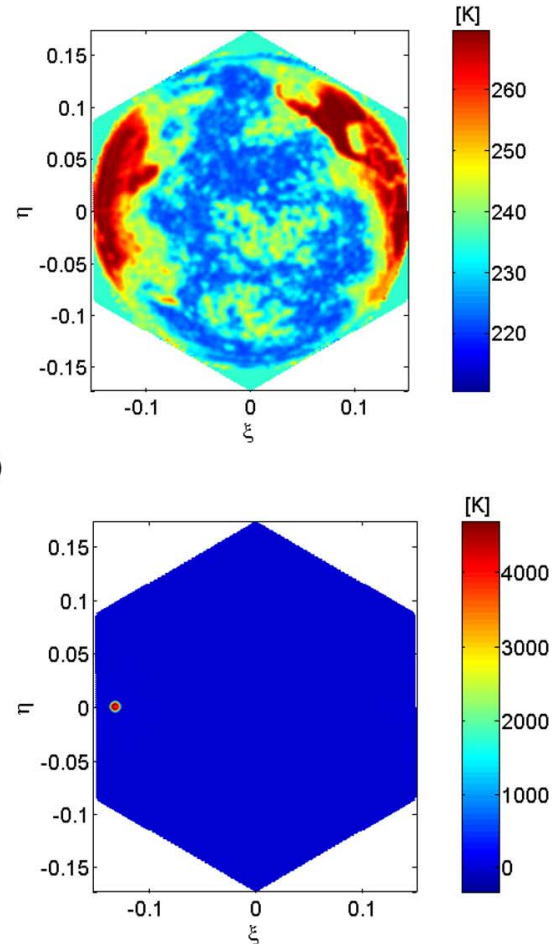

(c)

(f)

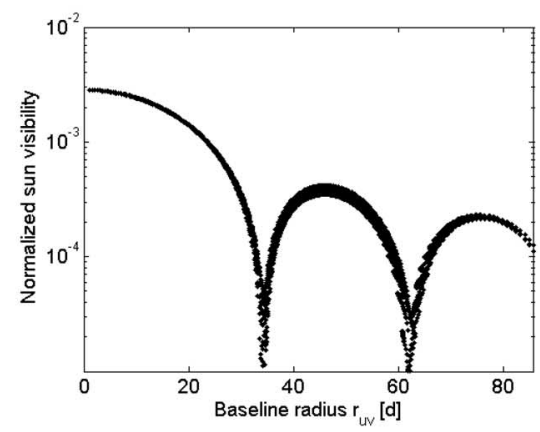

Fig. 1. (a) Test image AMSU-A channel number 3 as seen from GEO. (b) Estimated image in the hexagonal cell given by GeoSTAR UFoV Sky alias corrected only inside the Earth circle. (c) Error image due to discretization and truncation errors. The circle $r<0.1$ used to compute rms error is also shown. (d) Error image for a $0.025^{\circ}$ out-of-plane arm \#1 misalignment. (e) Retrieved image showing sun alias when the sun is at $\xi_{\text {sun }}=0.17, \eta_{\text {sun }}=0$. The Earth contribution is eliminated by differential imaging, and the sun alias corresponds to the alias image centered at $\left(\xi_{c 0}, \eta_{c 0}\right)=(-0.132,0)$, as computed in the example given in Section II. (f) Amplitude spectrum of sun normalized visibilities as a function of distance to $u-v$ plane center $\left(T_{\mathrm{sys}}=600 \mathrm{~K}\right)$. The relevance of images (e) and (f) is given in Section VII.

about $d=2.3 \mathrm{~cm}(3.825 \lambda$ at $50.3 \mathrm{GHz})$, the length of each arm of the "Y" would be about $2.3 \mathrm{~m}$. The complex cross correlation between the signals collected by any pair of antennas gives one sample of the so-called visibility function, which is the measure of a particular spatial harmonic. The "Y"-shaped distribution of the antennas gives the visibility samples over a hexagonal grid, which minimizes the number of elements required to cover the spatial frequency domain $u-v$ (antenna separation measured in wavelengths). The equation that relates the sample of the visibility function (in units of kelvin), which are measured between antennas $k$ and $j$ at a single polarization, to the brightness temperature is given by [2], [9]

$$
\begin{aligned}
V_{k j}\left(u_{k j}, v_{k j}\right)= & \frac{1}{\sqrt{\Omega_{k} \Omega_{j}}} \iint_{\xi^{2}+\eta^{2}} \frac{T_{B}(\xi, \eta)-T_{\mathrm{rec}}}{\sqrt{1-\xi^{2}-\eta^{2}}} \\
& \cdot F_{n k}(\xi, \eta) F_{n j}^{*}(\xi, \eta) \widetilde{r}_{k j}\left(-\frac{u_{k j} \xi+v_{k j} \eta}{f_{0}}\right) \\
& \cdot \exp \left(-\mathrm{j} 2 \pi\left(u_{k j} \xi+v_{k j} \eta\right)\right) d \xi d \eta
\end{aligned}
$$

where $T_{B}$ is the brightness temperature, $T_{\text {rec }}$ is the receiver's physical temperature (assumed equal in all elements), $F_{n k}$ and $F_{n j}$ are the normalized antenna copolar voltage patterns, $\Omega_{k, j}$ is the antenna's solid angle, $\widetilde{r}_{k j}$ is the fringe-washing function $\left(\widetilde{r}_{k j} \cong 1\right.$ for GeoSTAR [6]), $f_{0}=c / \lambda$ is the center frequency, $\xi=\sin \theta \cos \phi$ and $\eta=\sin \theta \sin \phi$ are the direction cosines, and $u_{k j}$ and $v_{k j}$ are the antenna separation measured in wavelengths (baseline). The contribution of $T_{\text {rec }}$ to the visibility samples is negligible in the GeoSTAR prototype due to the low antenna coupling and the large antenna separation [9]. In the case of equal antenna patterns, the so-called modified brightness temperature is defined as

$$
T_{M}(\xi, \eta)=\frac{T_{B}(\xi, \eta)}{\sqrt{1-\xi^{2}-\eta^{2}}} \frac{\left|F_{n}(\xi, \eta)\right|^{2}}{\Omega^{2}} .
$$

Now, following the procedure described in [7], the visibility equation can be rewritten in its discrete form as

$$
\begin{aligned}
V_{k j}=\Delta s \cdot \sum_{m=1}^{N} & \sum_{n=1}^{N} T_{M}\left(\xi_{m n}, \eta_{m n}\right) \\
\cdot & e^{-j 2 \pi\left(u_{k j} \xi_{m n}+v_{k j} \eta_{m n}+w_{k j} \sqrt{1-\xi_{m n}^{2}-\eta_{m n}^{2}}\right)}
\end{aligned}
$$

where $\Delta s$ is the pixel area in the $(\xi, \eta)$ domain. The sampling points (spatial frequencies) are computed as

$$
u_{k j}=\frac{x_{k}-x_{j}}{\lambda} \quad v_{k j}=\frac{y_{k}-y_{j}}{\lambda} \quad w_{k j}=\frac{z_{k}-z_{j}}{\lambda}
$$

where the spatial coordinates $\left(x_{k}, y_{k}, z_{k}\right)$ and $\left(x_{j}, y_{j}, z_{j}\right)$ are antenna $k$ and $j$ phase centers. Note that an out-of-plane sampling coordinate $w_{k j}[10]$ has also been included in the discrete form of (1) to account for out-of-plane errors in the following sections. In a simplified form, (3) can be written as

$$
\mathbf{V}=\mathbf{G} \cdot \mathbf{T}_{\mathbf{M}}
$$


where $\mathbf{G}$ is the so-called G-matrix [7]. In the ideal case, the interferometric integral (1) becomes a Fourier transform, and it can be computed in its discrete form as a fast Fourier transform (FFT) [8].

The image selected to estimate the impact of array distortion is obtained from the Advanced Microwave Sounding Unit (AMSU-A) channel number $3(50.3 \mathrm{GHz})$ remapped from LEO observations to a GEO view [Fig. 1(a)], in which the sky has been set to $2.7 \mathrm{~K}$. The image is given in a $1000 \times 1000$ grid in the direction cosine domain, which gives a spatial resolution at boresight of $72 \mathrm{~km}$. To make G-matrix simulations compatible with Fourier analysis, the image has been interpolated to a hexagonal grid in the $(\xi, \eta)$ domain that is the reciprocal basis of the sampling domain $(u, v)$ [8]. In this case, the pixel area is $\Delta s=d^{2} \sqrt{3} / 2$, where $d$ is the nominal antenna separation within one arm (Fig. 2, top). The modified brightness temperature $T_{M}\left(\xi_{m n}, \eta_{m n}\right)$ is computed by taking into account the theoretical antenna patterns of the Potter antennas used in the ground demonstrator [6]. To minimize the number of antennas required for a given spatial resolution, the $u-v$ domain is undersampled: the real image is recovered at the center of the $(\xi, \eta)$ plane, but six aliases of the image appear around it, which are centered at [9]

$$
\left(\xi_{c n}, \eta_{c n}\right)=\frac{2}{d \sqrt{3}}\left[\cos \left(n \frac{\pi}{3}\right), \sin \left(n \frac{\pi}{3}\right)\right],
$$

The smallest spacing in the sampling grid is set to $d=3.825 \lambda$ to give GeoSTAR unambiguous field of view (UFoV) as the hexagonal cell shown in Fig. 1(b). The same hexagonal image is repeatedly attached to each of the six hexagon sides in Fig. 1(b). For instance, if we take into account the alias image given by $n=0$, this alias is centered at $\left(\xi_{c 0}, \eta_{c 0}\right)=(0.302,0)$. Since the Earth as seen from GEO is constrained to $|\xi|<0.15$, the antenna separation $d$ has been set so that the UFoV matches the Earth disk [Fig. 1(b)]. However, the actual image is constrained to the unit circle [Fig. 1(a)], and the sky from each of the six alias images is superposed on the main image. This effect can be removed provided that the brightness temperature of the sky is known.

To sum up, the direct case (from brightness temperature to visibility) is performed by means of the G-matrix when position errors are present and by FFT in the ideal case. The inverse procedure (from visibility to brightness temperature) is always performed by means of the inverse FFT. A routine to remove the effect of the sky is used in all the simulations in this paper.

\section{RADIOMETRIC ERROR DUE TO ARM Mis AlignMENT}

An example of the nominal Y-shaped array for $N_{\mathrm{el}}=8$ elements per arm is shown in Fig. 2 (middle). The elements are arranged in such a way that the three arm axes meet in an equilateral triangle. This is the so-called "staggered-Y" configuration, which is used to simplify the mechanical and electrical designs by eliminating the one odd center receiver [6].

As a first-order approach, arm distortion is modeled by means of an in-plane $(\beta)$ and an out-of-plane $(\alpha)$ phase offset in relation to its nominal position (Fig. 2, top). Arm distortion will likely be more complex. However, this approximation will
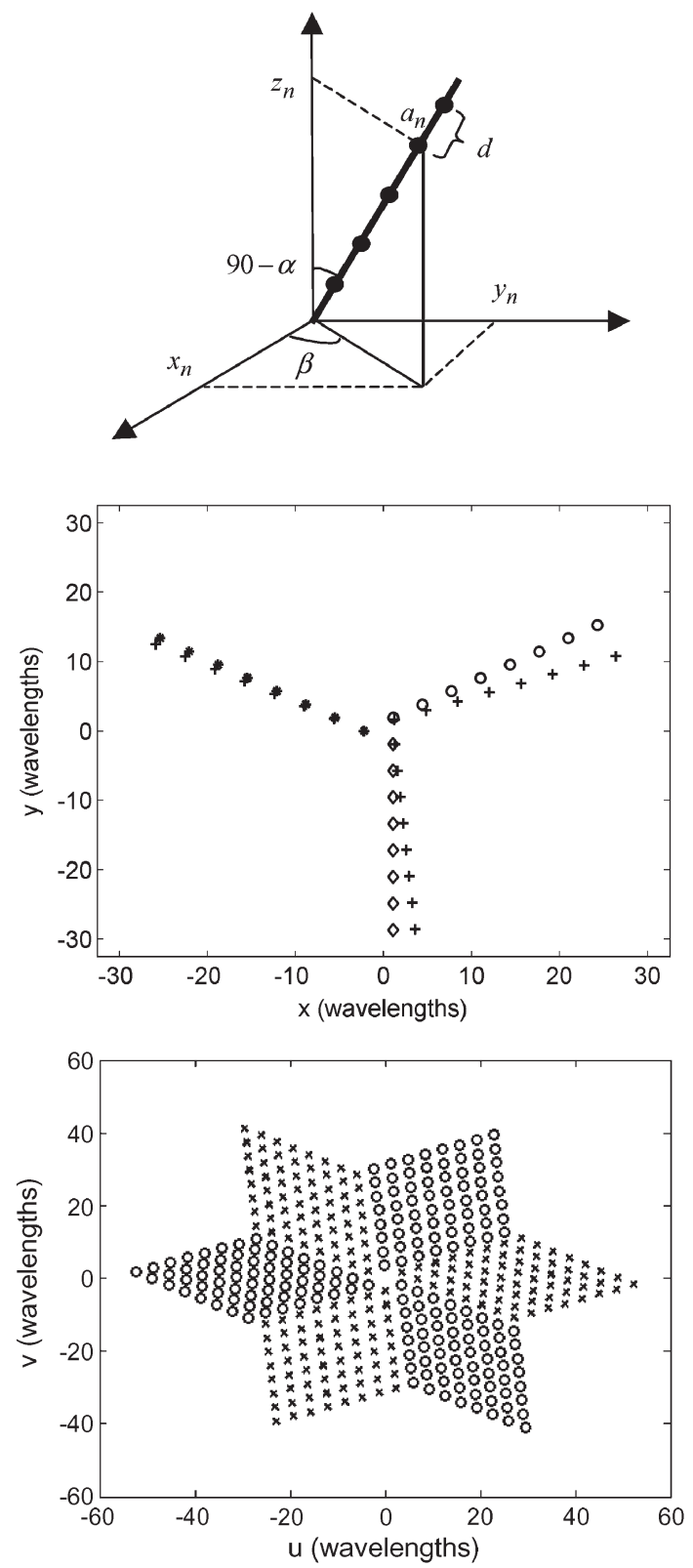

Fig. 2. (Top) Model of antenna distribution for an arm placed along $x$-axis with out-of-plane $(\alpha)$ and in-plane $(\beta)$ misalignment errors. (Middle) Example of antenna distribution for the nominal Y-shaped array $\left(N_{\mathrm{el}}=8\right)$ : $\operatorname{arm} \# 1(\diamond)$, $\# 2(*)$, and \#3 (०). The plus sign $(+)$ shows an example of arm misalignment: arm \#1, $5^{\circ} ; \# 2,-2^{\circ}$; and \#3, $-10^{\circ}$. (Bottom) Sampling grid in the $u-v$ domain for the distorted array: $(\mathrm{x})$ measured $(u, v)$ and $(\circ)$ symmetric $(-u,-v)$ baselines.

give us a good insight into the amount of distortion that can be tolerated to keep radiometric error within specifications. If we take into account an arm placed approximately along the $x$-direction, the coordinates of the $n$th antenna from the array center can be computed according to the parametric equation of the straight line as

$$
\left.\begin{array}{l}
x_{n}=a_{n} \cdot r+b_{x} \\
y_{n}=a_{n} \cdot s+b_{y} \\
z_{n}=a_{n} \cdot t+b_{z}
\end{array}\right\} .
$$

If the origin of coordinates is placed at the center of the array, and the staggered configuration of GeoSTAR is taken into 
account [6], the line coefficients are

$$
\left.\begin{array}{l}
a_{n}=\left(n-\frac{1}{2}\right) d \\
b_{x}=b_{z}=0 \\
b_{y}=-\frac{d}{2} \tan \left(30^{\circ}\right)
\end{array}\right\}
$$

where $d$ is the antenna separation expressed in wavelengths. The parameters $r, s$, and $t$ can be expressed as a function of the in-plane $(\beta)$ and out-of-plane $(\alpha)$ phase misalignment as

$$
\left.\begin{array}{l}
r=\sin \left(\frac{\pi}{2}-\alpha\right) \cos \beta \\
s=\sin \left(\frac{\pi}{2}-\alpha\right) \sin \beta \\
t=\cos \left(\frac{\pi}{2}-\alpha\right)
\end{array}\right\} .
$$

Now, each one of the three arms that comprise the Y-shaped array must be rotated an angle $\phi_{R i}$ from its initial position on the $x$-axis $\left(\phi_{R 1}=270^{\circ}, \phi_{R 2}=150^{\circ}\right.$, and $\left.\phi_{R 3}=30^{\circ}\right)$. After the rotation, spatial coordinates for a specific antenna $n$ placed along a particular arm $i(i=1,2,3)$ can be rewritten as

$$
\left.\begin{array}{l}
x_{n}^{i}=a_{n} \cdot r_{i} \cos \phi_{R i}-\left(a_{n} \cdot s_{i}+b_{y}\right) \sin \phi_{R i} \\
y_{n}^{i}=\left(a_{n} \cdot s_{i}+b_{y}\right) \cos \phi_{R i}+a_{n} \cdot r_{i} \sin \phi_{R i} \\
z_{n}^{i}=a_{n} \cdot t_{i}
\end{array}\right\} .
$$

Fig. 2 (middle) shows an example of antenna in-plane misalignment $\left(N_{\mathrm{el}}=8\right)$, and Fig. 2 (bottom) shows its associated sampling grid in the $u-v$ domain. Combining (4) and (10), all sampling coordinates $\left(u_{k j}, v_{k j}, w_{k j}\right)$ can be readily computed. Visibility samples are then calculated by means of the G-matrix [7]. If there is no arm misalignment $(\alpha=0, \beta=0), \mathbf{V}$ can be computed by means of a rectangular FFT [8]. In any case, once the visibility samples are calculated, an estimation of the brightness temperature map can be retrieved by means of an inverse FFT [8]. An error function $\Delta T\left(\xi_{m n}, \eta_{m n}\right)$ is defined as the difference between the original and the retrieved image. The mean and the standard deviation of $\Delta T\left(\xi_{m n}, \eta_{m n}\right)$ are computed inside a circle $r<0.1$, which corresponds to the $45^{\circ}$ incidence angle from GEO. In Fig. 1(a), for $N_{\mathrm{el}}=50$ antennas per arm, if the ideal case is taken into account, truncation and finite coverage yield a radiometric error $\sigma_{\Delta T}=0.19 \mathrm{~K}$ and negligible bias [Fig. 1(c)].

Fig. 3 shows the radiometric error increase in relation to the ideal case for in-plane (top) and out-of-plane (bottom) misalignment errors in arm \#1. The sensitivity to these errors is readily computed from the slope of the plots, which yields $S_{\alpha}^{\sigma_{T}}=15.8 \mathrm{~K} / \mathrm{deg}$ for out-of-plane misalignment and $S_{\beta}^{\sigma_{T}}=$ $0.9 \mathrm{~K} / \mathrm{deg}$ for in-plane misalignment errors. For instance, Fig. 1(d) shows $\Delta T\left(\xi_{m n}, \eta_{m n}\right)$ for an out-of-plane error $\alpha=$ $0.025^{\circ}$ in arm \#1, which yields $\sigma_{\Delta T}=0.4 \mathrm{~K}$. Simulations taking into account arms \#2 and \#3 yield similar results, while radiometric errors due to simultaneous misalignment in multiple arms have been found to add in an approximate quadratic sense (uncorrelated errors).

\section{RADIOMETRIC ERROR DUE TO RANDOM ANTENNA POSITION ERROR}

Random position errors of antenna phase centers can be simulated with a procedure similar to the one used in the
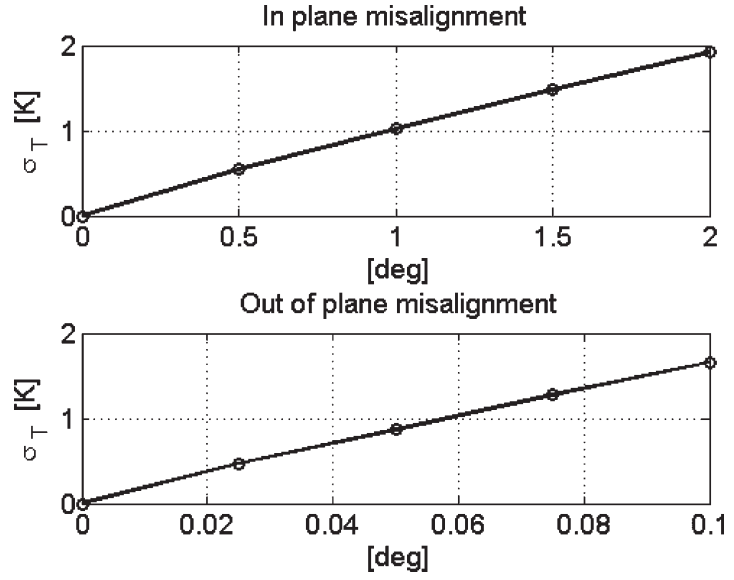

Fig. 3. Radiometric error due to in-plane (top) and out-of-plane (bottom) arm \#1 misalignment. The radiometric error is computed as the increase in relation to the ideal case [Fig. 1(c)].

previous section. Misalignment is not taken into account in (7) by setting $r=1$ and $s=t=0$. Then, a zero mean random error in wavelengths is added to each antenna coordinate as

$$
\left.\begin{array}{l}
x_{n}=a_{n}+b_{x}+\Delta x_{m} \\
y_{n}=\Delta y_{m} \\
z_{n}=\Delta z_{m}
\end{array}\right\}
$$

where $\Delta x_{m}$ and $\Delta y_{m}$ are in-plane along and normal arm positions errors, respectively. Both have equal standard deviation $\sigma_{x}=\sigma_{y} . \Delta z_{m}$ represents an out-of-plane position error with standard deviation $\sigma_{z}$. The impact of each error value ( $\sigma_{x}=\sigma_{y}$ or $\sigma_{z}$ ) is obtained out of 25 simulations. Position errors in antennas close to the center of the array have been found to produce a larger impact on radiometric performance. To better evaluate this feature, two types of error distribution along the arms are performed.

1) Uniform error: In this case, the statistics of antenna position errors among the 25 simulations is equal for all antennas within an arm.

2) Weighted error: In this case, the standard deviation of the position errors is weighted with the distance to the center of the array so as to give almost zero error for the first antenna and $2 \sigma_{x}=2 \sigma_{y}$ (or $2 \sigma_{z}$ ) for the antennas at the edge of each arm.

Fig. 4 shows the results for the four different cases. Since the vertical scale is the same for all plots, the horizontal scale indicates the relative sensitivity of these four different errors. Due to the large contribution of the shortest baselines, out-of-plane position errors with uniform distribution (top right) produce the largest radiometric error. The following sections will show that an out-of-plane error produces an almost constant phase error in the signal collected by each antenna. Since $\sigma_{\phi} \cong 2 \pi \sigma_{z} / \lambda$, the equivalent sensitivity to out-of-plane position errors can be computed as (Appendix)

$$
S_{\sigma_{z}}^{\Delta T} \cong 2 \pi S_{\sigma_{\phi}}^{\Delta T}[\mathrm{~K} / \mathrm{rad}]=444.6 K / \lambda .
$$

Note that position errors $\sigma_{z}$ are given in wavelengths. For comparison, Fig. 4 (top, right) also shows the radiometric error computed according to this formula (diamonds). Note that both 

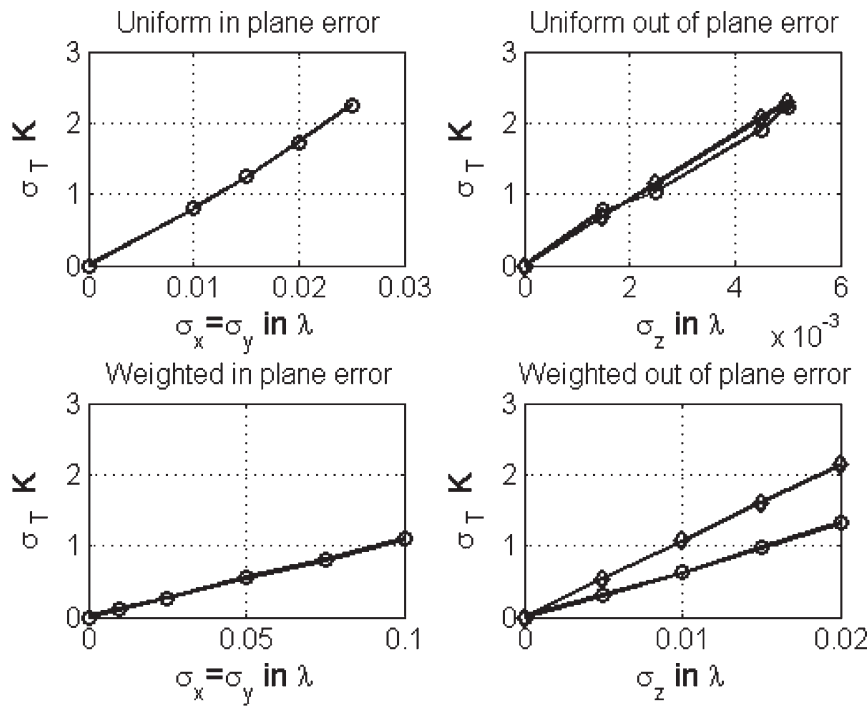

Fig. 4. Radiometric error due to in-plane and out-of-plane random $u-v$ position errors for uniformly and linearly weighted error distribution along the arms (circles). $N_{\mathrm{el}}=50$. The test image is shown in Fig. 1(a). The diamonds represent the impact of an equivalent out-of-plane path delay phase (Appendix).

results match quite well in spite of the fact that the procedure to perform the simulations has been completely different.

- To take into account the direction dependency of the error in the computation of visibility samples, out-ofplane position errors have been modeled by means of the G-matrix.

- Out-of-plane position errors have been approximated by means of a constant phase error in each antenna, which allowed us the use of FFT to perform the simulations (Appendix).

The in-plane position errors in Fig. 4 (top, left) are given as a function of the radius of uncertainty circle computed as

$$
\sigma_{r}=\sqrt{\sigma_{x}^{2}+\sigma_{y}^{2}}=\sqrt{2} \sigma_{x}
$$

As shown in Fig. 4 (bottom, diamonds), if the position errors are distributed with linearly increasing error toward the edge of the arms, their impact is much lower than in the uniform distribution case. This demonstrates, as expected, that distortion is dominated by position errors in the antennas close to the center of the array. The linearly weighted error case does not have a direct physical meaning. However, in the case that some redundant antennas are used in the array (see Appendix), the error distribution along the arm approximates the weighted distribution. In this case, the sensitivity to out-of-plane position errors when redundancy is available can be computed from the sensitivity to phase errors as (Appendix)

$$
S_{\sigma_{z}}^{\Delta T} \cong 2 \pi S_{\sigma_{\phi}}^{\Delta T}[\mathrm{~K} / \mathrm{rad}]=106.9 K / \lambda
$$

For comparison, Fig. 4 (bottom, right) shows the radiometric error computed according to the formula above (circles), which is close to the linearly weighted case (diamonds).
TABLE I

\begin{tabular}{|c|c|c|}
\hline Distortion mode & Sensitivity & $\begin{array}{l}\text { Distortion to give } \\
\qquad \sigma_{\Delta T}=0.25 \mathrm{~K}\end{array}$ \\
\hline In plane arm misalignment & $0.9 \mathrm{~K} / \mathrm{deg}$ & $\pm 0.28 \mathrm{deg}$ \\
\hline Out of plane arm misalignment & $15.8 \mathrm{~K} / \mathrm{deg}$ & $\pm 0.016 \mathrm{deg}$ \\
\hline $\begin{array}{l}\text { In plane uniform random } \\
\text { antenna position error }\end{array}$ & $89.4 \mathrm{~K} / \lambda$ & $\sigma_{x}=\sigma_{y}=0.0028 \lambda$ \\
\hline $\begin{array}{l}\text { Out of plane uniform random } \\
\text { antenna position error }\end{array}$ & $427.5 \mathrm{~K} / \lambda$ & $\sigma_{z}=0.0006 \lambda$ \\
\hline $\begin{array}{l}\text { In plane weighted random } \\
\text { antenna position error }\end{array}$ & $10.9 \mathrm{~K} / \lambda$ & $\sigma_{x}=\sigma_{y}=0.023 \lambda$ \\
\hline $\begin{array}{l}\text { Out of plane weighted random } \\
\text { antenna position error }\end{array}$ & $66.2 \mathrm{~K} / \lambda$ & $\sigma_{z}=0.0038$ \\
\hline Stretching error & Negligible & - \\
\hline
\end{tabular}

SUMMARY OF DISTORTION MODES

\section{IMPACT OF STRETCHING ERRORS}

Stretching errors occur because of thermal changes and gradients within the arms, which yield variations from the nominal antenna separation $d$. In a first approach, the actual antenna separation $d_{s}$ can be computed by taking into account a uniform temperature increase along one arm that gives a uniform antenna separation change

$$
d_{s}=d\left(1+\frac{s t}{10^{6}} \Delta T_{p h}\right) .
$$

Mechanical and thermal analysis predicts that the arm stretching coefficient is $s t=28 \mathrm{ppm} /{ }^{\circ} \mathrm{C}$ and that the physical temperature can be well constrained to $\pm 5^{\circ} \mathrm{C}$ (a quite conservative goal). In this case, if we take into account a worst case situation, e.g., $+5^{\circ}$ in arm $\# 1$ and $-5^{\circ}$ in arm \#3, simulations yield a $\sigma_{\Delta T}=0.011 \mathrm{~K}$ error in comparison to the ideal case. This effect can be considered negligible relative to the $1 \mathrm{~K}$ goal for the global error budget [11] and does not deserve further analysis at this point.

\section{Summary of Distortion Modes}

Table I gives a summary of the radiometric error sensitivity to the different distortion modes. Since the error budget establishes an overall $1-\mathrm{K}$ radiometric error, the table also gives the magnitude of each distortion mode to give $0.25-\mathrm{K}$ contribution to the error budget. This error is equal for all cases just for comparison reasons. It is found that in-plane distortion can be well constrained by mechanical design and thermal control. However, radiometric error presents a high sensitivity to outof-plane distortion, which would place quite tough design requirements. The next section is devoted to show that an external source allows to practically eliminate the impact of out-of-plane distortion (Fig. 5). The interested reader can find an alternative method to analyze array imperfections in [12].

\section{Correction of ARray Distortion}

As shown in Table I, radiometric error due to array distortion presents a high sensitivity to out-of-plane position errors, and some kind of correction will significantly ease array design. This section is devoted to analyzing the capability of a point 

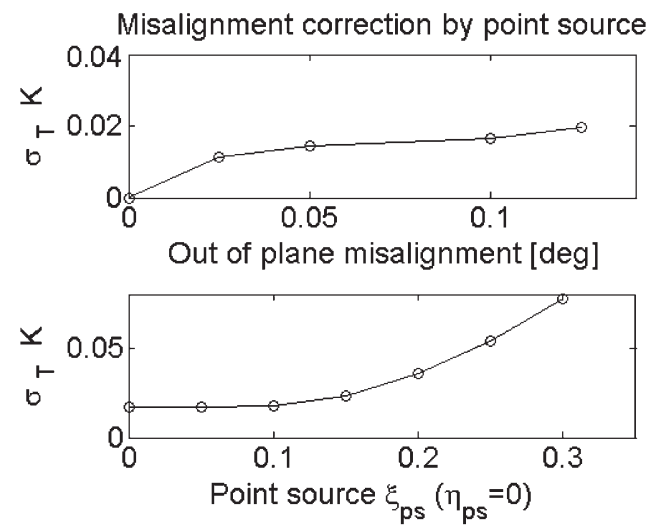

Fig. 5. Radiometric error due to out-of-plane arm misalignment after phase correction by means of a point source placed at boresight (top). Effect of the point source position for an out-of-plane misalignment $\alpha=0.1^{\circ}$ (bottom).

source to reduce the impact of out-of-plane errors. We assume that a point source with apparent modified brightness temperature $T_{\mathrm{ps}}$ placed at pixel $\left(\xi_{\mathrm{ps}}, \eta_{\mathrm{ps}}\right)$ is given by

$$
T_{M}\left(\xi_{m n}, \eta_{m n}\right)=T_{\mathrm{ps}} \delta\left(\xi_{m n}-\xi_{\mathrm{ps}}, \eta_{m n}-\eta_{\mathrm{ps}}\right)
$$

where $\delta$ is the 2-D Dirac delta. The point source visibility measured between antennas $k$ and $j$ is given by introducing (16) into (1) as

$$
V_{k j}^{\mathrm{ps}}=\frac{\sqrt{3}}{2} d^{2} T_{\mathrm{ps}} e^{-j 2 \pi\left(u_{k j} \xi_{\mathrm{ps}}+v_{k j} \eta_{\mathrm{ps}}\right)} e^{-j 2 \pi\left(w_{k j} \sqrt{1-\xi_{\mathrm{ps}}^{2}-\eta_{\mathrm{ps}}^{2}}\right)}
$$

where only the out-of-plane errors $w_{k j}$ have been taken into account $\left(\Delta u_{k j}, \Delta v_{k j} \approx 0\right)$. The point source image can be corrected from out-of-plane distortion by subtracting the phase error term from each visibility sample as

$$
\phi_{k j}^{\mathrm{pse}}=-2 \pi\left(w_{k j} \sqrt{1-\xi_{\mathrm{ps}}^{2}-\eta_{\mathrm{ps}}^{2}}\right) .
$$

In the case of an extended source of radiation such as the Earth, this correction cannot be performed because the term $\phi_{k j}^{\mathrm{pse}}$ is different for each pixel. However, taking into account that GeoSTAR UFoV is quite narrow and constrained to the Earth contour

$$
\xi_{m n}^{2}+\eta_{m n}^{2}<r_{\text {Earth }}^{2}
$$

$\left(r_{\text {Earth }}=0.15\right)$, the phase correction factor changes less than $1.1 \%$ within the field of view. Therefore, the estimated visibility when imaging the Earth $V_{k j}^{e}$ can be partially corrected from outof-plane errors as

$$
V_{k j}^{c}=V_{k j}^{e} e^{-j \phi_{k j}^{\mathrm{pse}}}
$$

where the phase correction term is estimated from the phase of the point source measured visibilities as

$$
\phi_{k j}^{\mathrm{pse}}=\arg \left\{V_{k j}^{\mathrm{ps}}\right\}+2 \pi\left(u_{k j} \xi_{\mathrm{ps}}+v_{k j} \eta_{\mathrm{ps}}\right) .
$$

Note that, if the point source is placed close to the center of the $\operatorname{UFoV}\left(\xi_{\mathrm{ps}}, \eta_{\mathrm{ps}} \approx 0\right)$, the correction factor is not affected by in-plane position errors $\left(\Delta u_{k j} \xi_{\mathrm{ps}}+\Delta v_{k j} \eta_{\mathrm{ps}} \cong 0\right)$, which in any case are a second-order effect (Fig. 4, top, left).

\section{A. Receiver Phase Calibration by Means of a Point Source}

This section presents a quantitative assessment of the phase calibration by means of a point source. It must be pointed out that this correction is very robust since it is capable of also dealing with instrumental phase errors [13]. In this more general case, the phase of the point source visibility can be written as

$$
\phi_{k j}^{\mathrm{pse}}=-2 \pi\left(w_{k j} \sqrt{1-\xi_{\mathrm{ps}}^{2}-\eta_{\mathrm{ps}}^{2}}\right)+\phi_{k}-\phi_{j}+\phi_{k j}+\phi_{n}
$$

where $\phi_{k}$ and $\phi_{j}$ are the phase terms related to each receiver, $\phi_{k j}$ is the phase of the fringe washing function, which depends on the discrepancies in filter frequency responses between different receivers (this term has been found negligible for the GeoSTAR demonstrator [6]), and finally $\phi_{n}$ is a random phase related to thermal noise. This last term can also be considered negligible since the point source can yield large values of correlation (large signal-to-noise ratio) and the integration time can be extended as necessary. Therefore, point source correction removes both path delay phases due to outof-plane misalignment and instrumental phase errors (assumed constant).

Fig. 5 (top) shows the radiometric error after calibration by means of a point source at boresight as a function of misalignment error. If the point source is moved away from the boresight, the radiometric error increases slightly since the phase correction term is less accurate for some areas within the UFoV (Fig. 5, bottom). However, even for point sources placed well beyond the Earth-sky border $(r>0.15)$, the correction error is below $0.05 \mathrm{~K}$. This suggests that the sun may also be used as a reference for phase calibration of the instrument.

\section{B. Sun as a Reference for Phase Calibration}

This section analyzes the suitability of the sun as a reference for phase calibration of the instrument. In a first approach, the phase of the visibility samples from the sun image depends exclusively on the position of the sun center as given by

$$
\phi_{k j}^{\mathrm{sun}}=-2 \pi\left(u \xi_{\mathrm{sun}}-v \eta_{\mathrm{sun}}\right) .
$$

However, the sun is not a point source, and the magnitude of the visibility samples is not constant. Fig. 1(f) shows the amplitude spectrum of the sun visibilities as a function of distance to the center of the $u-v$ plane

$$
r_{u v}=\sqrt{u^{2}+v^{2}} .
$$

The noise in the normalized correlations due to finite integration time is given by $\sigma_{r, i}=0.5 / \sqrt{B \tau_{\text {eff }}}$, where $\tau_{\text {eff }}=\tau / 2.46$ at a sampling rate twice the signal bandwidth. Since thermal noise is independent of the magnitude of the visibility sample, the signal-to-noise ratio of measured samples decreases due to the decay of the visibility function. As a conclusion, since most 
of the measured visibilities present a very low signal-to-noise ratio, the sun cannot be used as it was proposed for the point source. The next subsection presents a quantitative analysis of the phase calibration procedure by means of the sun.

\section{Alternative Calibration by Means of the Sun}

This section is devoted to develop an alternative phase calibration by means of the sun that overcomes the fact that it is not a point radio source. The Appendix shows that the radiometric error sensitivity to phase errors in relation to the image given in Fig. 1(a) is $S_{\sigma_{\phi}}^{\Delta T}=1.23 \mathrm{~K} / \mathrm{deg}$ for uniform phase error distribution along the arms and $S_{\sigma_{\phi}}^{\Delta T}=0.3 \mathrm{~K} / \mathrm{deg}$ in the case of a linearly weighted distribution (array with redundancy). Taking into account an overall radiometric error of $1 \mathrm{~K}$, if $0.25 \mathrm{~K}$ is assigned to phase errors, it must be constrained to $\sigma_{\phi}=0.2 \mathrm{deg}$ in the uniform case and $\sigma_{\phi}=0.8 \mathrm{deg}$ in the linearly weighted case.

An estimation of the phase error term, which includes both instrumental and path delay error phases, can be written as

$$
\hat{\phi}_{k j} \cong \phi_{k j}^{\mathrm{sun}}-2 \pi\left(u \xi_{\mathrm{sun}}+v \eta_{\mathrm{sun}}\right)=\phi_{k}-\phi_{j}
$$

where the thermal noise $\phi_{n}$ and the fringe-washing $\phi_{k j}$ terms have been neglected. Considering an instrument composed of $N_{r}$ receivers, the following set of equations can be established:

$$
\begin{gathered}
{\left[\begin{array}{c}
\hat{\phi}_{12} \\
\hat{\phi}_{13} \\
\ldots \\
\hat{\phi}_{1 N_{r}} \\
\hat{\phi}_{23} \\
\ldots \\
\hat{\phi}_{\left(N_{r}-1\right) N_{r}}
\end{array}\right]=\left[\begin{array}{ccccccc}
1 & -1 & 0 & 0 & 0 & \ldots & 0 \\
1 & 0 & -1 & 0 & 0 & \ldots & 0 \\
\ldots & \ldots & \ldots & \ldots & \ldots & \ldots & 0 \\
1 & 0 & 0 & \ldots & \ldots & \ldots & -1 \\
0 & 1 & -1 & 0 & \ldots & \ldots & 0 \\
\ldots & \ldots & \ldots & \ldots & \ldots & \ldots & \ldots \\
0 & 0 & \ldots & \ldots & 0 & 1 & -1
\end{array}\right]} \\
\\
\end{gathered}
$$

This can be written in a simplified form as

$$
\hat{\phi}_{\mathbf{k j}}=\mathbf{P} \cdot \phi_{\mathbf{k}}
$$

The number of visibility samples in the case that all possible correlation pairs between antennas are measured is given by $N_{v}=N_{r}\left(N_{r}-1\right) / 2$ (this includes the redundant antenna pairs within each single arm). Then, the size of the matrix $\mathbf{P}$ is $N_{v} \times N_{r}$. Although there are $N_{r}$ phases to retrieve, the rank of the matrix $\mathbf{P}$ is always $\left(N_{r}-1\right)$ because the system is formed by phase differences. This implies that we can set one of the channels as a reference and assign a zero phase to it (which is equivalent to subtracting its phase from the phase of all other channels). The reference phase must be eliminated from
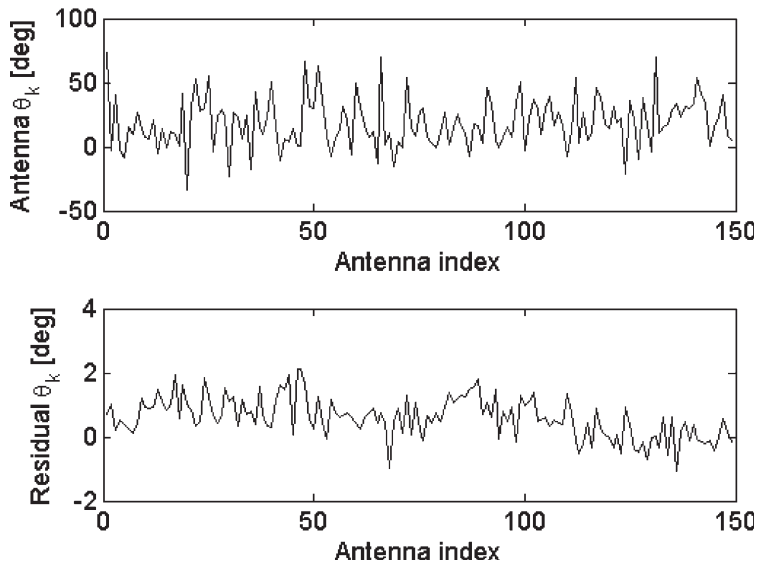

Fig. 6. (Top) Constant phase error term assigned to each antenna $\left(N_{\mathrm{el}}=50\right)$, which includes out-of-plane path delay phase. (Bottom) Residual error in the retrieved phase using the sun as reference. The visibility samples are corrupted by thermal noise (1-s integration time), and only the samples where $r_{u v}<$ $10 d$ are used. Calibration error is reduced to a negligible value by averaging consecutive 1-s sun calibrations.

the column vector $\phi_{\mathbf{k}}$, and its related column from the system matrix $\mathbf{P}$ must also be eliminated. Now the size of the new matrix $\mathbf{P}$ is $N_{v} \times\left(N_{r}-1\right)$, and its rank remains $N_{r}-1$. This allows the retrieval of $N_{r}-1$ unknown phases from $N_{v}$ visibility samples. However, this procedure would yield large errors in the estimation of phases because the phase $\hat{\phi}_{k j}$ from measured visibilities presents a large error in most of the visibility samples due to very poor signal-to-noise ratio. Fortunately, the system can be solved in a least-squares sense even in the case that only the equations related to visibility samples with large signal-to-noise ratio are used.

As an example, we can take into account the sun placed at $(\xi, \eta)=(0.1700,0)$ in Fig. 1(a), which gives an image of the sun alias placed in $(\xi, \eta)=(-0.1320,0)$ in Fig. 1(e). For a system with $N_{\mathrm{el}}=50$ elements per arm, the number of receivers is $N_{r}=150$, and the number of visibility samples is $N_{v}=11.175$. To perform the simulation, the following steps are performed.

- The maximum phase drift due to sun movement for any visibility sample with $r_{u v}<10 d$ is limited to $1.5^{\circ}$. This sets the integration time to $1 \mathrm{~s}$.

- A thermal noise with standard deviation $\sigma_{r, i}=8 \cdot 10^{-5}$ is added to the real and imaginary parts of the normalized correlation. This corresponds to an integration time of $t=1 \mathrm{~s}$.

- We have selected the visibility samples that satisfy the condition $r_{u v}<10 d$. In this case, the magnitude of the remaining visibility samples is larger than $|\mu|=2,6 \cdot 10^{-3}$ [Fig. 1(f)], which gives a signal-to-noise ratio larger than 20 and a random phase $\phi_{n}$ with a standard variation lower than $\sigma_{\phi_{n}}=1.7^{\circ}$.

- Now, all rows in $\mathbf{P}$ where $r_{u v}>10 d$ are removed. To simulate a constant phase error in each antenna, a random phase is assigned to each one (Fig. 6, top). The phase of the measured visibility samples is then computed by adding the phase term $\phi_{k}-\phi_{j}$ to the ideal sun visibilities $V_{k j}$. 
The unknown phases $\phi_{\mathrm{k}}$ are now retrieved by solving the thinned system of equations in a least-squares sense. Fig. 6 (bottom) shows the residual phase error after retrieving the phase terms by means of the procedure presented so far. Since the mean error in the estimated phases is $0.29^{\circ}$ and $\sigma_{\phi}=0.69^{\circ}$, the retrieval procedure has proved to be quite robust. Now, the residual phase error in the retrieved phases can be reduced to a negligible value by averaging consecutive 1-s sun calibrations. Once the individual phases related to each antenna are retrieved, all visibility samples measured when imaging the Earth $V_{k j}^{e}$ can be corrected as

$$
V_{k j}^{c}=V_{k j}^{e} e^{-j\left(\phi_{k}-\phi_{j}\right)} .
$$

As a conclusion, using the sun as a phase reference has proved to be a quite robust procedure to phase calibrate the instrument as long as only the visibility samples with higher signal-tonoise ratio are used. As a drawback, the sun does not provide a continuous phase calibration of the instrument since it can only be performed close to eclipse. That is, to eliminate the contribution of the Earth, this technique requires two images: one when the sun is seen in the sky close to eclipse; and a second one when the sun is hidden behind the Earth disk. It must be pointed out that an artificial point source placed on an appropriate location on the Earth, which could be switched on and off as required, would provide a very good alternative to the sun.

\section{CONCLUSION}

GeoSTAR array distortion has been analyzed, and the outof-plane antenna position errors have been found to be the dominant source of radiometric error. Since the major contribution to the error budget comes from the errors in the shorter baselines (low spatial harmonics), the inclusion of a small set of additional antennas in the center of the array significantly reduces the impact of such errors. These additional antennas are used to measure the so-called redundant visibilities (the same baseline is measured by different antenna pairs), which are required to mitigate the effect of antenna failure [14], [15] and to improve system performance by averaging the error in the shorter baselines. Since GeoSTAR UFoV is very narrow, the out-of-plane antenna position errors are well approximated by a constant phase error term assigned to each antenna, which can be corrected by means of a point source. The calibration procedure presents a quite low sensitivity to the position of the point source, and some preliminary results have also shown that the sun, under certain constraints, can be used as a reference for phase calibration.

\section{APPENDIX}

\section{ImPaCt of CONSTANT PHASE ERRORS IN THE ANTENNAS}

This Appendix is devoted to assessing the radiometric error given by a constant phase error in each antenna. To perform the simulations, a constant phase error is assigned to each single receiver with a zero mean normal distribution within the set of

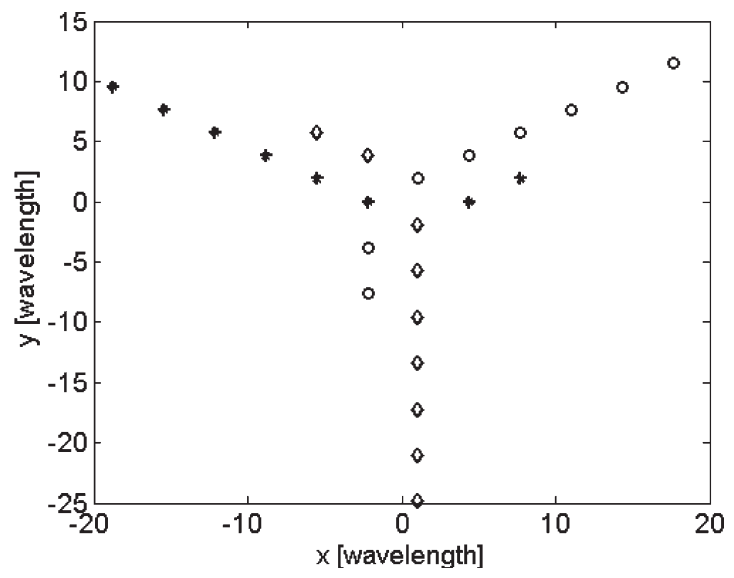

Fig. 7. GeoSTAR redundant topology for $N_{\mathrm{el}}=8$ and three redundant antennas per arm: arms \#1 $(\diamond), \# 2(*)$, and \#3 (०). Antennas with the same symbol are assigned to the same arm so as to form antenna pairs and compute correlations [6].

$N_{r}$ receivers. The estimated visibility measured by receivers $k$ and $j$ when $\theta_{k}$ and $\theta_{j}$ phase errors are present can be written as

$$
\hat{V}_{k j}=V_{k j} e^{j\left(\theta_{k}-\theta_{j}\right)}
$$

where $V_{k j}$ is the ideal visibility sample, which is computed by means of the FFT [8]. The reference scene used to compute $V_{k j}$ is given in Fig. 1(a). An estimation of the original brightness temperature image is then retrieved by applying an inverse FFT to the corrupted visibility samples $\hat{V}_{k j}$. Now, an error function $\Delta T(\xi, \eta)$ can be defined as the temperature difference between the original and retrieved images. Radiometric error is then calculated as the standard deviation of $\Delta T(\xi, \eta)$ within the circle $\sqrt{\xi^{2}+\eta^{2}}<0.1$ (incidence angle lower than $45^{\circ}$ ). The radiometric error $\sigma_{\Delta T}$ related to each phase error distribution with standard deviation $\sigma_{\phi}$ is computed by performing 100 sets of simulations. That is, $\sigma_{\Delta T}$ is computed as the average value of the standard deviation of $\Delta T(\xi, \eta)$ for 100 different sets of phase error distributions with the same standard deviation $\sigma_{\phi}$ along the arms.

The radiometric error due to phase errors is computed as the error increase (in a quadratic sense) in relation to the zero phase error case. It should be pointed out that even in the case that phase errors are not present, there is a small radiometric error $\sigma_{\Delta T}=0.19 \mathrm{~K}$ due to discretization and truncation of the visibility function (instrument with $N_{\mathrm{el}}=50$ elements per arm). The sensitivity to phase errors has been found to be $S_{\sigma_{\phi}}^{\Delta T}=1.23 \mathrm{~K} / \mathrm{deg}$. However, it is known that the radiometric error is mainly contributed by phase errors in the shorter baselines due to the fact that it contains a larger amount of signal energy [15]. This suggests that its impact can be reduced by including additional antennas close to the center of the array (Fig. 7). These give the so-called redundant visibilities (same antenna spacing formed by different antenna pairs), which are required to improve instrument robustness [14]. In this case, the error distribution along the arms is not uniform since the shorter baselines are redundant and the error averages. Fig. 7 shows an example of a redundant topology. Now, proceeding in a similar 
way as done before, the radiometric error sensitivity to phase errors, when redundancy is available, is $S_{\sigma_{\phi}}^{\Delta T}=0.3 \mathrm{~K} / \mathrm{deg}$ (20 redundant elements per arm), which gives a value about four times lower than the nonredundant case.

\section{ACKNOWLEDGMENT}

Simulations were based on the simulator InRadSim, which was developed by Polytechnic University of Catalonia (Spanish Research and EU Feder Project TEC2005-06863-C02-01/ TCM, "Técnicas interferométricas, polarimétricas y biestáticas para sistemas de teledetección por microondas") and has been adapted to the GeoSTAR configuration.

\section{REFERENCES}

[1] B. Lambrigtsen, W. Wilson, A. Tanner, T. Gaier, C. Ruf, and J. Piepmeier, "GeoSTAR-A microwave sounder for geostationary satellites," in Proc. IEEE IGARSS, 2004, vol. 2, pp. 777-780.

[2] C. S. Ruf, C. T. Swift, A. B. Tanner, and D. M. Le Vine, "Interferometric synthetic aperture microwave radiometry for the remote sensing of the Earth," IEEE Trans. Geosci. Remote Sens., vol. 26, no. 5, pp. 597-611, Sep. 1988.

[3] D. M. Le Vine, C. T. Swift, and M. Haken, "Development of the synthetic aperture microwave radiometer, ESTAR," IEEE Trans. Geosci. Remote Sens., vol. 39, no. 1, pp. 199-202, Jan. 2001.

[4] M. Martín-Neira and J. M. Goutoule, "A two-dimensional aperturesynthesis radiometer for soil moisture and ocean salinity observations," ESA Bull., no. 92, pp. 95-104, Nov. 1997.

[5] P. Silvestrin, M. Berger, Y. Kerr, and J. Font, "ESA's second Earth explorer opportunity mission: The soil moisture and ocean salinity mission-SMOS," IEEE Geosci. Remote Sens. Newslett., no. 118, pp. 1114, Mar. 2001.

[6] A. B. Tanner, S. T. Brown, S. J. Dinardo, T. M. Gaier, P. P. Kangaslahti, B. H. Lambrigtsen, W. J. Wilson, J. R. Piepmeier, C. S. Ruf, S. M. Gross, B. H. Lim, S. Musko, and S. Rogacki, "Initial results of the GeoSTAR prototype (geosynchronous synthetic thinned array radiometer)," in Proc. IEEE Aerosp. Conf., Big Sky, MT, Mar. 4-11, 2006. DOI 10.1109/AERO.2006.1655814.

[7] A. B. Tanner and C. T. Swift, "Calibration of a synthetic aperture radiometer," IEEE Trans. Geosci. Remote Sens., vol. 31, no. 1, pp. 257-267, Jan. 1993.

[8] A. Camps, J. Bara, I. Corbella, and F. Torres, "The processing of hexagonally sampled signals with standard rectangular techniques: Application to 2-D large aperture synthesis interferometric radiometers," IEEE Trans. Geosci. Remote Sens., vol. 35, no. 1, pp. 183-190, Jan. 1997.

[9] I. Corbella, N. Duffo, M. Vall-llossera, A. Camps, and F. Torres, "The visibility function in interferometric aperture synthesis radiometry," IEEE Trans. Geosci. Remote Sens., vol. 42, no. 8, pp. 1677-1682, Aug. 2004.

[10] M. Peichel, H. Suess, and M. Suess, "Microwave imaging of the brightness temperature distribution of extended areas in the near and far field using two-dimensional aperture synthesis with high spatial resolution," Radio Sci., vol. 33, no. 3, pp. 781-801, May/Jun. 1998.

[11] A. B. Tanner, W. Wilson, B. Lambrigsten, S. Dinardo, S. Brown, P. Kangaslahti, T. Gaier, C. Ruf, S. Gross, B. Lim, S. Musko, and S. Rogacki, "Initial results of the geosynchronous synthetic thinned array radiometer (GeoSTAR)," in Proc. IGARSS, 2006, pp. 3968-3971.

[12] M. Peichl and H. Suess, "Error analysis of sparse passive synthesis radiometers on UAV platforms," in Proc. SPIE Conf. - Passive MillimeterWave Imaging Technology VIII, Orlando, FL, Mar. 30-31, 2005, pp. 1-10.

[13] F. Torres, A. Camps, J. Bará, and I. Corbella, "Impact of receiver errors on the radiometric resolution of large 2D aperture synthesis radiometers. Study applied to MIRAS," Radio Sci., vol. 32, no. 2, pp. 629-641, Mar./Apr. 1997.

[14] M. Vall-llossera, N. Duffo, A. Camps, I. Corbella, F. Torres, and J. Bara, "Reliability analysis in aperture synthesis interferometric radiometers: Application to L-band microwave imaging radiometer with aperture synthesis instrument," Radio Sci., vol. 36, no. 1, pp. 107-117, Jan. 2001.

[15] F. Torres, A. B. Tanner, S. T. Brown, and B. H. Lambrigsten, "Robust array configuration for a microwave interferometric radiometer: Application to the GeoSTAR project," IEEE Geosci. Remote Sens. Lett., vol. 4, no. 1, pp. 97-101, Jan. 2007.

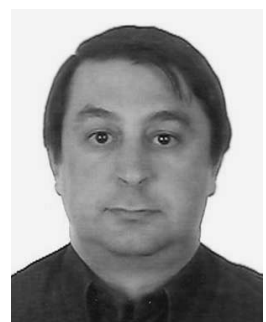

Francesc Torres (M'96-SM'06) received the Ingeniero and Doctor Ingeniero degrees in telecommunication engineering from the Polytechnic University of Catalonia (UPC), Barcelona, Spain, in 1988 and 1992, respectively.

From 1988 to 1989 , he was a Research Assistant in the RF System Division, European Space Agency, Noordwijk, The Netherlands, where he was devoted to microwave device testing and characterization. Since 1989, he has been with the Antenna Microwave Radar Group, UPC, where he is currently an Associate Professor. From August 2005 to July 2006, he held a sabbatical stage at the Jet Propulsion Laboratory, California Institute of Technology, Pasadena, CA. His main research interests are focused on the design and testing of microwave systems and subsystems, and also on interferometric radiometers devoted to Earth observation.

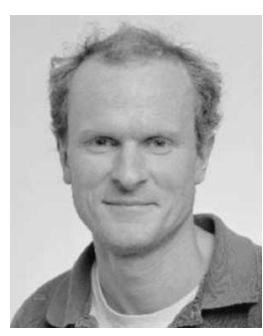

Alan B. Tanner received the B.S. and Ph.D. degrees from the University of Massachusetts, Amherst, in 1984 and 1989, respectively.

He is currently a Microwave Systems Engineer at the Jet Propulsion Laboratory (JPL), California Institute of Technology, Pasadena, CA. His work at JPL has focused on the design and calibration of radiometers and radar scatterometers for remote sensing.

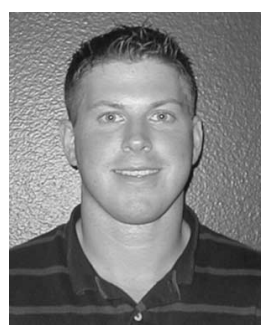

Shannon T. Brown (M'05) received the B.S. degree in meteorology from Pennsylvania State University, University Park, and the M.S. and Ph.D. degrees, in 2005, from the University of Michigan (UMich), Ann Arbor.

In 2005, he joined the Microwave Advanced Systems Section, NASA Jet Propulsion Laboratory, Pasadena, CA, as a member of the engineering staff. He has also been involved with the spaceborne Topex and Jason Microwave Radiometers, the WindSat Polarimetric Radiometer, and the Jason follow-on Advanced Microwave Radiometer. His research interests include microwave radiometer calibration, geophysical algorithm development for both passive and active sensors, and cloud and precipitation science.

Dr. Brown was a recipient of the NASA Group Achievement Award in 2004 for his contribution to the UMich/Goddard Space Flight Center Lightweight Rainfall Radiometer.

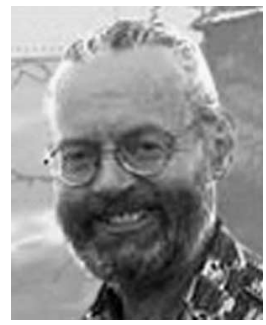

Bjorn H. Lambrigsten joined the Jet Propulsion Laboratory (JPL) in 1982. He specializes in atmospheric remote sensing and related research. $\mathrm{He}$ is the GeoSTAR Principal Investigator and leads a number of other efforts as well, including hurricanerelated research. He is a member of the NPOESS Preparatory Project science team, is the Microwave Instrument Scientist for the Atmospheric Infrared Sounder project, and leads the AIRS Atmospheric Science Group at JPL. 